\title{
Uma Nova Metodologia para Detecção de Falhas em Sistemas Dinâmicos Não Lineares Baseada em Análise Externa Fuzzy
}

\author{
Luís M. M. Torres* Fernanda S. Sousa ** \\ Maria K. M. Monteiro ${ }^{* * *}$ Selmo E. Rodrigues Júnior* \\ * Instituto Federal de Educação, Ciência e Tecnologia do Maranhão / \\ Campus Imperatriz (e-mail: luis.torres@ifma.edu.br). \\ ** Instituto Federal de Educação, Ciência e Tecnologia do Maranhão / \\ Campus Imperatriz (e-mail: fernandasousa3@hotmail.com) \\ *** Instituto Federal de Educação, Ciência e Tecnologia do Maranhão / \\ Campus Imperatriz (e-mail: mkelianemacedo@gmail.com)
}

\begin{abstract}
In this work, a new methodology based on the External Analysis with fuzzy model is proposed, for the monitoring of nonlinear processes that operate in multiple modes of operation. The set-point of the control loop and the manipulated variable were defined as external variables and the main variable is composed of the process variable. A set of training data was used in the estimation of the regression coefficients of the fuzzy model. In order to obtain the rules of the fuzzy model the antecedent parameters were obtained by the clustering algorithm FCM, and the consequent by least squares method. Computational results were obtained from the application of the proposed methodology in a closed loop system with a PI controller, making a comparison between the false positive and false negative rates with other external analysis approaches existent in the literature, in order to illustrate the efficiency of the proposed methodology.

Resumo: Neste trabalho, é proposta uma nova metodologia baseada na Análise Externa com modelo fuzzy, para o monitoramento de processos não lineares que atuam em múltiplos modos de operação. A referência da malha de controle e a ação de controle foram definidas como variáveis externas e a variável principal é composta pela variável de processo. Um conjunto de dados de treinamento foi utilizado na estimação dos coeficientes de regressão para o modelo fuzzy. Para a obtenção das regras do modelo fuzzy os parâmetros do antecedente foram estimados pelo algoritmo de agrupamento FCM, e o consequente pelo método dos mínimos quadrados. Resultados computacionais foram obtidos a partir da aplicação da metodologia proposta em um sistema em malha fechada com um controlador PI, fazendo um comparativo entre as taxas de falsos positivos e de falsos negativos com outras abordagens de análise externa existentes na literatura, com o intuito de ilustrar a eficiência da metodologia proposta.
\end{abstract}

Keywords: Fault Detection; Nonlinear Dynamical Systems; External Analysis; Fuzzy Systems; Takagi-Sugeno Model.

Palavras-chaves: Detecção de Falhas; Sistemas Dinâmicos Não Lineares; Análise Externa; Sistemas Fuzzy; Modelo Takagi-Sugeno.

\section{INTRODUÇÃO}

Atualmente, a complexidade de sistemas industriais e da robótica tais como não linearidades, incertezas, variações paramétricas e grandes volumes de dados (big data), trouxe novos desafios, maiores exigências e novas direções de pesquisas dando origem a métodos de monitoramento de processos baseados em dados. Geralmente, as técnicas tradicionais para a detecção e o diagnóstico de falhas são limitadas a processos que atuam em um único modo de operação, sendo portanto, necessário utilizar técnicas de detecção mais sofisticadas, dentre as quais destaca-se a Análise Externa proposta por Kano et al. (2004).

A análise externa fornece uma abordagem avançada para o monitoramento de processos com múltiplos modos de operação, excluindo-se a necessidade de definir um número limitado de regiões, apresentando vantagens de implementação que trabalham na detecção de defeitos de processo, mau funcionamento de equipamentos ou outros eventos (Wang et al., 2019).

Nesse sentido, o modelo fuzzy Takagi-Sugeno (Takagi and Sugeno, 1985) também é uma alternativa eficiente para solucionar problemas complexos em sistemas industriais, consolidando-se como uma metodologia bem sucedida em diversas aplicações. Em Salgado et al. (2017), é proposto um algoritmo de agrupamento fuzzy híbrido para a obtenção de modelos fuzzy Takagi-Sugeno. O principal objetivo desta abordagem é trabalhar com séries temporais com características variantes no tempo. Em Rotondo et al. (2015), é apresentado um estudo das analogias e conecções entre 
modelos lineares variantes no tempo (LPV) e modelos fuzzy Takagi-Sugeno; e são propostas duas metodologias para a geração automática de tais modelos. Em Filho and de Oliveira Serra (2017), é apresentada uma metodologia recursiva para identificação neuro-fuzzy evolutiva aplicada a sistemas não lineares com dinâmica não estacionaria na presença de ruído.

Dessa maneira, neste trabalho é proposta uma nova metodologia para detecção de falhas em sistemas dinâmicos não lineares baseada em análise externa com modelo fuzzy. Para tanto, foi realizado o estudo comparativo de duas metodologias de análise externa amplamente encontradas na literatura: análise externa com modelo simples (Kano et al., 2004) e análise externa com múltiplos modelos (FARIA et al., 2017); com a metodologia proposta neste trabalho. Tendo-se como principais objetivos a detecção de falhas em sistemas dinâmicos não lineares, bem como, avaliar o desempenho das metodologias propostas através de suas aplicações na supervisão do sistema dinâmico não linear simulado.

Este artigo está organizado da seguinte forma: na seção II, é apresentada o método de detecção via variáveis externas com as metodologias propostas. A seção III trata dos resultados computacionais das metodologias aplicadas ao processo baseado em uma malha de controle. Finalmente, na seção IV, as conclusões e trabalhos futuros são discutidos.

\section{DETECÇÃO DE FALHAS EM SISTEMAS DINÂMICOS NÃO LINEARES}

Nos processos industriais, as mudanças nas condições de operação de um sistema devem ser distinguidos de falhas ou mau funcionamento. Kano et al. (2004) propuseram um método promissor de detecção de falhas baseado em análise externa. Nesta seção, abordagens deste método com modelo simples, múltiplos modelos e a proposta de modelo fuzzy serão apresentadas, na perspectiva de comparar o desempenho das metodologias.

\subsection{Análise externa}

A análise externa foi originalmente desenvolvida como parte da análise de componentes principais restrita (CPCA) para combinar recursos de análise de regressão e PCA, no intuito de melhorar o desempenho do PCA usando informações externas (Kano et al., 2004),(Takane and Shibayama, 1991).

As variáveis utilizadas para monitoramento de um sistema podem ser classificadas como variáveis externas ou variáveis principais. As variáveis externas representam condições operacionais, tais como uma taxa de fluxo de alimentação e um ponto de referência. Enquanto que as variáveis principais são afetadas por variáveis externas e outros distúrbios não mensurados. As mudanças nas variáveis externas e sua influência nas variáveis principais podem ser diferenciadas das falhas ou do mau funcionamento, visto que os dados de operação das variáveis principais, de acordo com Kano et al. (2004), podem ser explicadas pelas variáveis externas através de um modelo de regressão.

Considerando uma matriz de dados $\mathbf{X} \in R^{k \times m}$, onde $k$ e $m$ são os números de amostras e variáveis, respectivamente, cada variável é considerada normalizada. Quando $m_{g}$ de $m$ variáveis são classificadas como variáveis externas e $m_{h}=\left(m-m_{g}\right)$ são variáveis principais, a matriz de dados $\mathbf{X}$ é descrita como:

$$
\mathbf{X}=\left[\begin{array}{ll}
\mathbf{H} & \mathbf{G}
\end{array}\right]
$$

onde $\mathbf{G}$ consiste em variáveis externas e $\mathbf{H}$ consiste em variáveis principais. Na análise de regressão linear múltipla pode-se usar as variáveis externas e variáveis principais como entradas e saídas, respectivamente. A matriz de coeficiente de regressão $\mathbf{C}$ é dada como:

$$
\mathbf{C}=\left(\mathbf{G}^{T} \mathbf{G}\right)^{-1} \mathbf{G}^{T} \mathbf{H}
$$

e a matriz de resíduos, que é a parte decomposta das variáveis principais que não depende das variáveis externas, pode ser representada por:

$$
\mathbf{E}=\mathbf{H}-\mathbf{G C}
$$

Quando a dinâmica do processo não pode ser ignorada, a influência de alterações em variáveis externas não pode ser removida dos dados de operação usando a análise externa estática. Nesse caso, um modelo dinâmico pode ser construído usando medições passadas de variáveis externas como entradas, uma vez que os dados principais no tempo $t, h(t)$, são afetados por dados externos no passado, $g(t-$ $1), g(t-2), \ldots$ Assim, a matriz de dados principais $\mathbf{H}$ e a matriz de dados externos $\mathbf{G}$ são modificadas da seguinte forma:

$$
\begin{gathered}
\mathbf{H}=\mathbf{H}_{\mathbf{0}}=\left[\begin{array}{c}
h(s) \\
\vdots \\
h(k-1) \\
h(k)
\end{array}\right] \\
\mathbf{G}=\left[\begin{array}{ll}
\mathbf{G}_{\mathbf{0}} & \mathbf{G}_{\mathbf{1}} \\
\ldots \mathbf{G}_{\mathbf{s}-\mathbf{1}}
\end{array}\right] \\
\mathbf{G}_{\mathbf{i}}=\left[\begin{array}{c}
g(s-i) \\
\vdots \\
g(k-1-i) \\
g(k-i)
\end{array}\right]
\end{gathered}
$$

onde $s$ é o tamanho da janela de amostras. Ou seja, dados externos no total de $s$ pontos de amostragem, $g(t), g(t-1), \ldots, g(t-s+1)$, são usados para estimar os dados principais $h(t)$. O parâmetro $s$ deve ser grande o suficiente para remover a influência de variáveis externas das variáveis principais.

\subsection{Análise externa com múltiplos modelos}

A análise externa aplicada em sistemas não lineares apresenta limitações quando apenas um modelo é utilizado para representar as diversas regiões de operações existentes. Diante de tais limitações FARIA et al. (2017) propôs uma metodologia de análise externa que considera múltiplos modelos, assumindo o efeito de não linearidades.

Dado o conjunto de dados de treinamento $X \in R^{n_{x}}(m+p)$, constituído de $n$ amostras contendo as $p$ variáveis externas 
e as $m$ variáveis principais. $\mathrm{O}$ algoritmo de classificação $\mathrm{k}$ means, proposto por Arthur and Vassilvitskii (2007), foi utilizado para agrupar os modelos de regressão $c$, onde é permitido escolher o número desejado de agrupamentos. Ressalta-se que outros métodos de agrupamento podem ser utilizados, como o algoritmo k-nearest neighbors (KNN), $k$-medians clustering, dentre outros.

Os coeficientes dos modelos de regressão $C_{k} \operatorname{com} k=$ $1, \ldots, c$, podem ser calculados usando as matrizes $G_{k}$ e $H_{k}$ obtidas para cada modelo, ou seja:

$$
C_{k}=\left(G_{k}^{\mathrm{T}} G_{k}\right)^{-1} G_{k}^{\mathrm{T}} H_{k}
$$

A matriz de resíduos de cada modelo é:

$$
\mathrm{E}_{\mathrm{k}}=\mathbf{H}_{\mathrm{k}}-\mathrm{G}_{\mathrm{k}} \mathrm{C}_{\mathrm{k}}
$$

Os resíduos de cada modelo de Análise Externa são utilizados para calcular a média e desvio padrão, empregados no treinamento do modelo PCA. A média e o desvio padrão são armazenados para serem utilizados na normalização de novas amostras durante a fase de monitoramento de falhas.

Definidos os $c$ modelos a partir dos dados de treinamento, faz-se necessário o uso de um classificador que indique qual modelo melhor representa a nova amostra de dados, como a classificação por Mínima Distância Euclidiana e por Distância de Mahalanobis. Adotando, a distância euclidiana dada uma nova amostra de dados associada a cada agrupamento é calculada por:

$$
D=\sqrt{\sum_{i=1}^{n}\left(\text { centros }-G_{i}\right)^{2}},
$$

onde a menor distância indica o modelo a ser utilizado para o monitoramento.

Após a escolha do modelo que melhor representa a nova amostra de dados, pode-se calcular o resíduo usando o modelo selecionado:

$$
\mathbf{E}=\mathbf{H}-\mathbf{G C}_{\mathbf{k}},
$$

Posteriormente, é realizada a normalização do resíduo correspondente ao modelo e o teste estatístico do resíduo normalizado.

\subsection{Análise externa fuzzy}

O modelo fuzzy Takagi-Sugeno (TS), originalmente proposto por Takagi and Sugeno (1985), consiste em uma base de regras IF-THEN. Os antecedentes de cada regra partem de um subconjunto das variáveis do modelo de um conjunto fuzzy e o consequente é uma expressão funcional simples. O sistema de inferência fuzzy TS é por natureza um aproximador universal de funções (Zeng and Singh Madan, 1994), por essa razão é proposta a utilização de um modelo fuzzy TS para a análise externa de sistemas dinâmicos não lineares.

No sistema de inferência fuzzy TS, a $\left(i^{(i=1,2, \ldots, L)}\right)$-ésima regra, sem perda de generalidade, possui a seguinte estrutura (Babuska, 1998):

$$
\begin{aligned}
& R^{(i)}: S E x_{1} \text { é } A_{1}^{i} E \ldots E x_{n} \text { é } A_{n}^{i} \text { ENTÃO } \\
& H^{i}=G C^{i}
\end{aligned}
$$

onde $x_{1}, x_{2}, \ldots, x_{n} \in R^{n}$ são os componentes da matriz de dados $\mathbf{X}$ (antecedente) e $H_{1}, H_{2}, \ldots, H_{m} \in R^{m}$ são as variáveis principais (consequente), em que $C^{i}$ é o coeficiente de regressão para cada regra. $R^{i}$ indica a $i$ ésima regra, e $L$ é o número de regras na base da regra. $A^{i}$ é o conjunto fuzzy antecedente da $i$-ésima regra, definida por uma função de pertinência:

$$
\mu_{A^{i}}(\mathrm{X}): \mathrm{R}^{\mathrm{n}} \rightarrow[0,1]
$$

O grau de ativação $h^{i}$ para a regra $R^{i}$, é dado por:

$$
h^{i}(\mathrm{X})=\mu_{\mathrm{A}_{1}^{\mathrm{i}}} \wedge \mu_{\mathrm{A}_{2}^{\mathrm{i}}} \wedge \ldots \wedge \mu_{\mathrm{A}_{\mathrm{n}}^{\mathrm{i}}}
$$

onde $\wedge$ é um operador de conjunção norma-t (Klir and Yuan, 1995). O grau de ativação normalizado para a regra $R^{i}$, é dado por:

$$
\lambda^{i}(\mathrm{X})=\frac{\mathrm{h}^{\mathrm{i}}(\mathrm{X})}{\sum_{\mathrm{r}=1}^{\mathrm{L}} \mathrm{h}_{\mathrm{r}}(\mathrm{X})}
$$

onde

$$
\sum_{r=1}^{L} h_{r}(\mathrm{X})>0
$$

$\operatorname{com} h_{r}(\mathrm{X})>0, i=1,2, \ldots, L$, esta normalização implica em:

$$
\sum_{i=1}^{L} \lambda(\mathrm{X})=1
$$

No sistema de inferência fuzzy TS, a resposta é uma soma ponderada dos parâmetros do consequente, como segue:

$$
\tilde{H}=\sum_{i=1}^{L} \lambda^{i} G C^{i}
$$

A estimação das funções de pertinência do antecedente do modelo fuzzy TS são obtidas em batelada a partir de um conjunto de dados experimentais usando o método de agrupamento fuzzy C-means (FCM) proposto por Bezdek et al. (1984). Os dados experimentais são observados através dos sinais de entrada e saída do sistema dinâmico. Cada observação consiste em $\mathrm{n}$ variáveis medidas, agrupadas em um vetor coluna $z_{k}=\left[z_{1 k}, z_{2 k}, \ldots, z_{n k}\right]^{\mathrm{T}}$, $z_{k} \in R^{n}$. Um conjunto de $n$ observações é expresso por $Z=z_{k} \mid k=1,2, \ldots, N$, onde $Z$ é a matriz de dados $n \times N$, representada como segue:

$$
Z=\left[\begin{array}{cccc}
z_{11} & z_{12} & \cdots & z_{1 N} \\
z_{21} & z_{22} & \cdots & 0 \\
\vdots & \vdots & \ddots & \vdots \\
z_{n 1} & z_{n 2} & \cdots & z_{n N}
\end{array}\right]
$$


onde as colunas são chamadas de padrões ou objetos e as linhas são chamadas de características ou atributos.

Uma partição c-fuzzy de $Z$ é uma família de grupos $\left\{A^{i} \mid i=1,2, \cdots, c\right\}$, em que o grau de pertinência que o $k$-ésimo ponto de dados possui em $A^{i}$ é dado pela seguinte notação:

$$
\mu_{k}^{i}=\mu_{A^{i}}\left(z_{k}\right) \in[0,1],
$$

sendo que a soma de todos os valores de pertinência para um dado ponto de dados em todos os agrupamentos deve ser unitária:

$$
\sum_{i=1}^{c} \mu_{k}^{i}=1
$$

para todo $k=1,2, \ldots, n$. Não podem haver grupos vazios, tampouco nenhum grupo pode conter todos os pontos de dados:

$$
0<\sum_{k=1}^{n} \mu_{k}^{i}<\eta .
$$

para todo $i$, onde $2 \leq c<N$, é um inteiro.

O espaço de particionamento fuzzy para $Z$ é o conjunto

$$
\begin{aligned}
& M_{f c}=\left\{U \in R^{c \times N} \mid \mu_{k}^{i} \in[0,1], \forall i, k ; \sum_{i=1}^{c} \mu_{k}^{i}=1, \forall k ;\right. \\
& \left.0<\sum_{k=1}^{N} \mu_{k}^{i}<N, \forall i\right\}
\end{aligned}
$$

onde a $i$-ésima linha da matriz de particionamento fuzzy $U$ contém valores das $i$-ésima funções de pertinências do subconjunto fuzzy $A^{i}$ de $Z$.

O objetivo é encontrar $U=\left[\mu_{k}^{i}\right] \in M_{f_{c}}$ e o vetor de protótipos de agrupamentos (centros), $V=\left[v_{1}, v_{2}, \ldots, v_{c}\right]$, com $v_{i} \in R^{n}$ tal que

$$
J(Z, U, V)=\sum_{i=1}^{c} \sum_{k=1}^{N}\left(\mu_{k}^{i}\right)^{m} D_{i k A}^{2}
$$

é minimizado, onde $m \in[1, \infty]$ é um expoente de ponderação que determina o grau de fuzzificação dos agrupamentos resultantes, e

$$
D_{i k A}^{2}=\left\|z_{k}-v_{i}\right\|_{A}^{2}=\left(z_{k}-v_{i}\right)^{\mathrm{T}} A\left(z_{k}-v_{i}\right)
$$

é uma norma de distância Euclidiana.

Os passos para implementação do algoritmo de agrupamento FCM são os seguintes citado nas referências Bezdek et al. (1984) e Wang (1997):

\section{Algoritmo Fuzzy C-means:}

Passo 1.Para o conjunto de dados $X=\left\{x_{1}, \ldots, x_{n}\right\}$, $x_{i} \in R^{P}$, determinando $c \in\{2,3, \ldots, n-1\}, m(1, \infty)$ e inicializar $U^{(O)} \in M_{f c}$.

Passo 2. Na iteração $l, l=0,1,2, \ldots$, calcule os vetores c means

$$
v_{i}^{l}=\frac{\sum_{k=1}^{n}\left(\mu_{i k}^{l}\right)^{m} x_{k}}{\sum_{k=1}^{n}\left(\mu_{i k}^{l}\right)^{m}}, 1 \leq i \leq c .
$$

Passo 3. Atualize $U^{(l)}=\left[\mu_{i k}^{(l)}\right]$ para $U^{(l+1)}=\left[\mu_{i k}^{(l+1)}\right]$ usando

$$
\begin{array}{rl}
D_{i k A}^{2} & =\left(z_{k}-v_{i}^{(l)}\right)^{\mathrm{T}} A\left(z_{k}-v_{i}^{(l)}\right), \\
1 \leq i \leq c, 1 \leq k \leq N & 1 \\
\mu_{i}^{l} & =\frac{1}{\sum_{j=1}^{c}\left(D_{i k A} / D_{j k A}\right)^{2 / m-1}}
\end{array}
$$

Passo 4. Se $\left|U^{(l+1)}-U^{(l)}\right|<\epsilon$, pare; caso contrário, ajuste $l=l+1$ e retorne ao Passo 2 .

O consequente do modelo fuzzy foi obtido a partir da (17), sendo representado na seguinte forma:

$$
H=\mathbf{\Lambda}^{i} \mathbf{G} C^{i}
$$

onde, $H$ é o vetor de saída do sistema dinâmico, $\mathbf{G}$ é a matriz de regressão, $\Lambda^{i}$ é a matriz de ponderação diagonal da $i$-ésima regra e $C^{i}$ é o vetor de coeficientes de regressão dos submodelos. O vetor de coeficientes de regressão pode ser obtido em batelada usando o método dos mínimos quadrados (Åström and Wittenmark, 2008), como a seguir:

$$
C^{i}=\left[\mathbf{G}^{\mathrm{T}} \boldsymbol{\Lambda}^{i} \mathbf{G}\right]^{-1} \mathbf{G}^{\mathrm{T}} \boldsymbol{\Lambda}^{i} H
$$

A formulação da (29) diz respeito a estimação local dos parâmetros do consequente, onde são estimados os parâmetros de cada submodelo separadamente.

Obtido $C^{i}$ e aplicando-o na (17) para determinar a saída estimada do modelo, pode-se encontrar os resíduos do seguinte modo:

$$
\mathbf{E}=\tilde{\mathbf{H}}-\mathbf{H}
$$

\subsection{Metodologia de Monitoramento}

Para o monitoramento do sistema é utilizada um limite de controle dado pela estatística $T^{2}$ de Hotelling. Tal limite estatístico com nível de confiança $\alpha$ foi definido utilizando uma distribuição $F_{\alpha}$ de Fischer-Snedecor (Mood, 1963), e é dado por

$$
T_{\alpha}^{2}=\frac{a\left(n^{2}-1\right)}{n(n-a)} F_{\alpha}(a, n-a)
$$

onde $\alpha$ é o nível de confiança, geralmente $5 \%$, e $n$ e $n-a$ os graus de liberdade, sendo $n$ o número de amostras e $a$ o número de variáveis principais.

O processo de monitoramento consiste nos seguintes passos:

1: Obter um modelo de análise externa (modelo simples, múltiplos modelos ou modelo fuzzy) a partir de um conjunto de dados que abranja toda a região de operação do processo a ser monitorado. 
2: Calcular a média e o desvio padrão dos resíduos $\mathbf{E}$ dos modelos obtidos no Passo 1.

3: Obter uma nova amostra do sistema em operação e calcular o resíduo utilizando o modelo de análise externa.

4: Padronizar os resíduo calculado utilizando a média e o desvio padrão dos resíduos obtidos na etapa de identificação.

5: Comparar o valor padronizado do resíduo com o limite de controle e tomar a decisão:

- Se $E_{k} \leq T_{\alpha}^{2}$ : Sistema sem presença de falha(s).

- Se $E_{k}>T_{\alpha}^{2}$ : Sistema na presença de falha(s).

\section{6: Retornar ao Passo 3.}

\section{RESULTADOS COMPUTACIONAIS}

Para a análise da metodologia proposta neste trabalho, foi utilizado via simulação computacional um sistema em malha fechada com um controlador PI, ilustrado na Figura 1, onde o sinal de saída $y(t)$ é a variável principal, e a ação de controle $u(t)$ e a referência $r(t)$ são as variáveis externas.

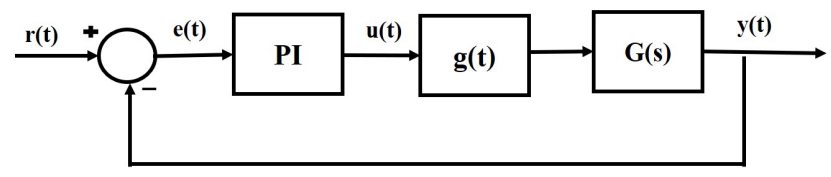

Figura 1. Diagrama em blocos do sistema em malha fechada.

A função de transferência em malha-aberta do sistema é dada por:

$$
G(s)=\frac{1}{(s+1)}
$$

com ganho em malha aberta de 4 para uma ação de controle no intervalo $[0,10]$ e mudando para 1 , quando a ação de controle assume valores maiores que 10, conforme (32):

$$
g(t)=\left\{\begin{array}{cc}
4 u(t), & 0 \leq u(t) \leq 10 \\
u(t)+30, & u(t) \geq 10
\end{array}\right.
$$

O sistema foi simulado em malha fechada com um controlador PI com ganhos proporcional e integral de 0,5626 e 4,4667, respectivamente.

Na primeira etapa da simulação, obteve-se o conjunto de dados de treinamento com 5000 amostras, sendo acrescido 10 unidades à referência a cada 500 amostras de dados. Os dados foram normalizados dentro da região de operação.

Os dados de treinamento foram utilizados na estimação dos coeficientes de regressão $\mathbf{C}$ para os três modelos: simples, múltiplos e fuzzy. Na etapa da estimação dos múltiplos modelos foi utilizado o algoritmo de agrupamento de classificação k-means com $c=4$ e norma euclidiana, no qual para cada agrupamento foi estimado os coeficientes dos modelos de regressão $C_{k} \operatorname{com} k=1, \ldots, c$, . Para o modelo fuzzy, a identificação em batelada foi realizada de acordo com a estrutura do modelo (11) para quatro regras, onde os parâmetros do antecedente foram obtidos
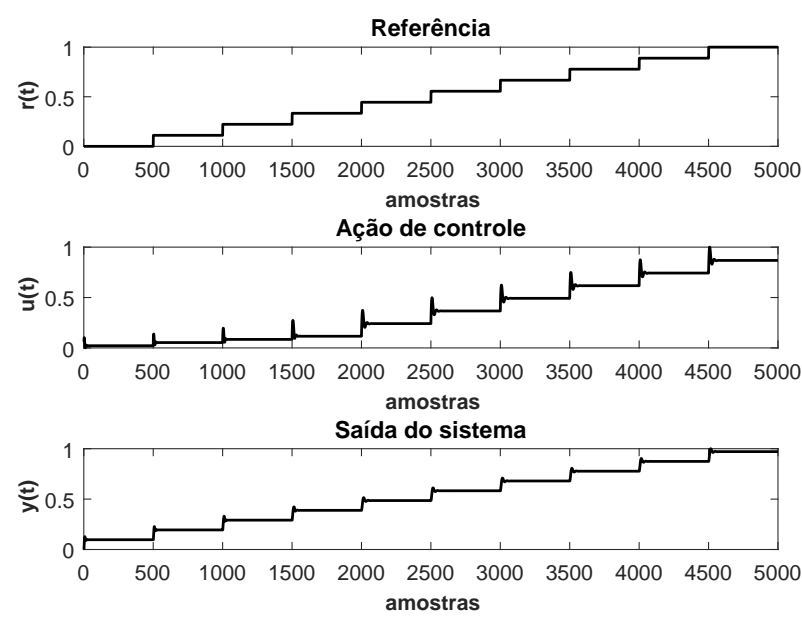

Figura 2. Conjunto de dados utilizado para o treinamento dos modelos baseados em análise externa.

pelo algoritmo de agrupamento FCM e os do consequente, vetor de coeficientes de regressão dos submodelos $C^{i}$, pelo método dos mínimos quadrados. Para todos os modelos foi utilizado $s=10$.
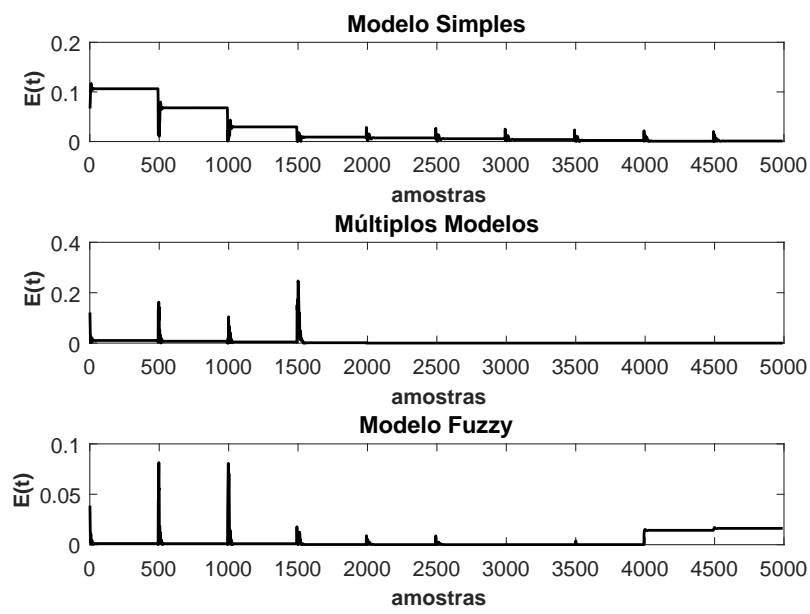

Figura 3. Resíduos dos modelos de análise externa.

\subsection{Falsos Positivos}

Inicialmente, na avaliação dos três modelos considerouse as taxas de falsos positivos (FP), onde FP pode ser definido como a porcentagem de registros falsos quando não existem falhas. A taxa de FP é calculada da seguinte maneira:

$$
F P=\frac{N L}{N T}
$$

onde NL é o número de amostras acima do limite estatístico sem a ocorrência de falha e NT é o número de amostras total sem a ocorrência de falha (Fernandes, 2018).

Para a realização dos testes foram feitas 1000 simulações com 5000 amostras de dados cada, sem a presença de 
falhas, como exemplificado na Figura 4. Nessas simulações, as referências foram geradas aleatoriamente dentro dos limites de operação do sistema.
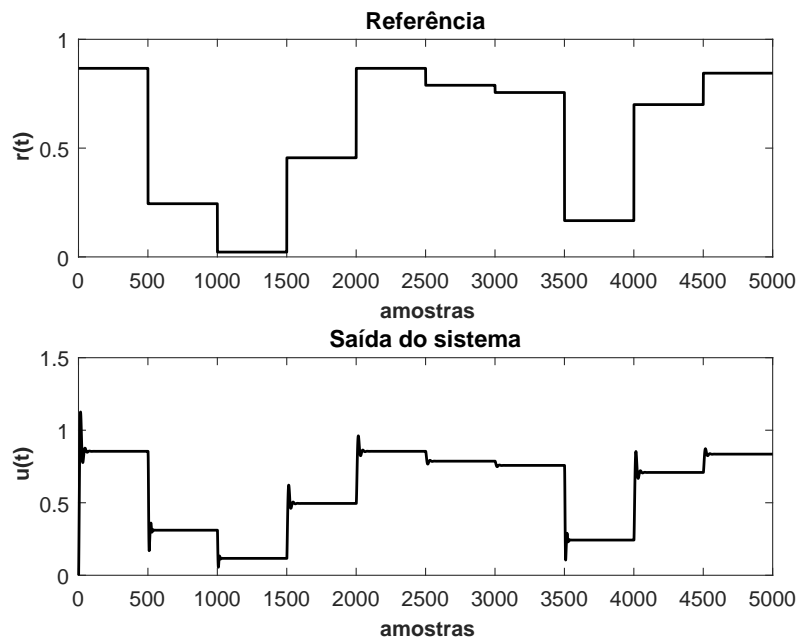

Figura 4. Exemplo de simulação utilizada para os testes de Falsos Positivos.

Na Tabela 1, são apresentados os resultados para os testes de FP, onde foram analisadas a quantidade de FP obtidos pelas três metodologias para as 1000 simulações realizadas. O modelo fuzzy proposto, mostrou-se superior aos demais na análise de FP, uma vez que os valores da média e do desvio padrão foram significativamente menores do que os valores encontrados para as outras metodologias.

\subsection{Falsos Negativos}

Posteriormente, na avaliação dos três modelos considerouse as taxas de falsos negativos $(\mathrm{FN})$, onde $\mathrm{FN}$ pode ser definido como a porcentagem de registros falsos de ausência de falhas quando existem falhas. As taxas de FN foram determinadas do seguinte modo:

$$
F N=\frac{N P F-N P D}{N P F}
$$

onde NPF se refere ao número de amostras após a ocorrência da falha e NPD ao número de amostras após a detecção da falha. Uma baixa taxa de FN indica sensibilidade do método, isto é, a capacidade do método de detectar falhas incipientes (Fernandes, 2018).

Para realização dos testes foram feitas 1000 simulações com 1000 amostras de dados cada, com a presença de falhas. Nessas simulações, as referências foram geradas aleatoriamente dentro dos limites de operação do sistema. Foram adicionados três tipos falhas de maneira aleatória, todas com a mesma probabilidade de ocorrerem. As falhas simuladas foram:

1- Diminuição de $20 \%$ no ganho integral;

2- Deslocamento do polo de malha aberta de $s=1$ para $s=2$;

3- Redução de $20 \%$ no ganho do sistema.

$\mathrm{Na}$ Figura 5 é ilustrada a resposta do sistema com a ocorrências das três falhas possíveis.
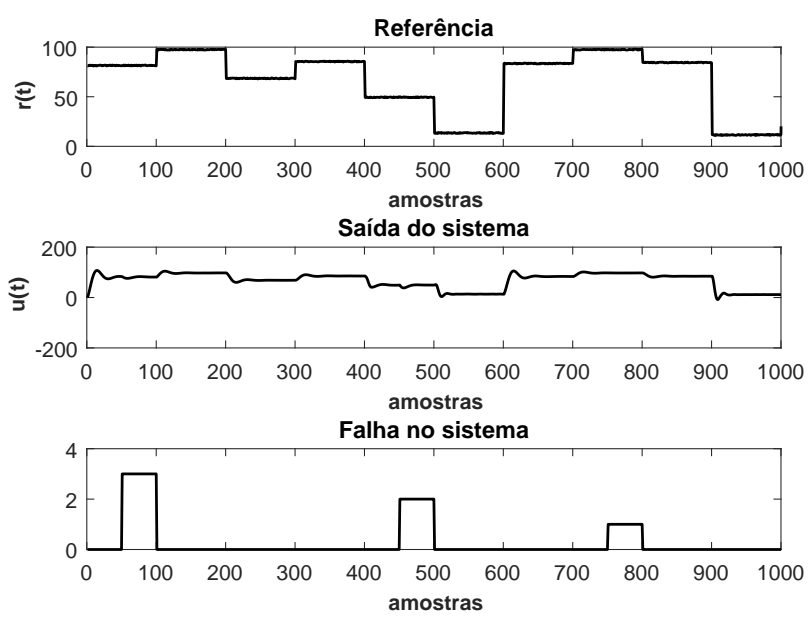

Figura 5. Exemplo de resposta do sistema na ocorrência das três possíveis falhas.

Na Tabela 2 são apresentados os resultados para os testes de FN, onde foram analisadas a quantidade de FN obtidos pelas três metodologias para as 1000 simulações realizadas. Neste caso, verificou-se um desempenho similar entre a metodologia de múltiplos modelos e modelo fuzzy, visto que não houveram diferenças significativas entre os valores da média e do desvio padrão.

\section{CONSIDERAÇÕES FINAIS}

Neste artigo, foi proposta uma nova metodologia para a detecção de falhas em sistemas dinâmicos não lineares baseada na análise externa com modelo fuzzy, utilizando a natureza de aproximador universal de funções do modelo fuzzy TS.

Os resultados obtidos com a metodologia proposta foram satisfatórios em comparação às outras metodologias já existentes na literatura, pois na análise dos testes de FP o modelo fuzzy obteve melhores resultados em relação às outras metodologias e resultados semelhantes na análise de FN. Por conseguinte, demonstra a eficiência e aplicabilidade da metodologia proposta neste trabalho.

A partir dos resultados obtidos neste trabalho, podem ser consideradas as seguintes propostas de trabalho futuro:

- Aplicação da metodologia em sistemas dinâmico não lineares multivariáveis;

- Utilização de outros algoritmos de agrupamento fuzzy;

- Aplicação em outros benchmarks existentes na literatura e/ou sistemas industriais.

\section{AGRADECIMENTOS}

Os autores agradecem ao IFMA pelo fomento a este trabalho.

\section{REFERÊNCIAS}

Arthur, D. and Vassilvitskii, S. (2007). K-means++: The advantages of careful seeding. SODA '07: Proceedings 
Tabela 1. Resultados obtidos para os testes de Falsos Positivos.

\begin{tabular}{ccccc} 
& Média & Desvio Padrão & Máximo & Mínimo \\
\hline Modelo Simples & 15,5626 & 11,3368 & 59,8 & $\mathbf{0}$ \\
Múltiplos Modelos & 6,5036 & 6,4116 & 31,52 & 0,12 \\
Modelo Fuzzy & $\mathbf{1 , 6 4 7 4}$ & $\mathbf{0 , 4 4 9 6}$ & $\mathbf{3 , 0}$ & 0,14 \\
\hline
\end{tabular}

Tabela 2. Resultados obtidos para os testes de Falsos Negativos.

\begin{tabular}{ccccc} 
& Média & Desvio Padrão & Máximo & Mínimo \\
\hline Modelo Simples & 10,0231 & 30,1434 & 100 & 0,01 \\
Múltiplos Modelos & 4,0138 & $\mathbf{1 9 , 6 9 1 8}$ & 100 & 0,01 \\
Modelo Fuzzy & $\mathbf{4 , 0 1 3 1}$ & 19,6919 & 100 & 0,01 \\
\hline
\end{tabular}

of the Eighteenth Annual ACMSIAM Symposium on Discrete Algorithms, 1027-1035.

Åström, K. and Wittenmark, B. (2008). Adaptive Control: Second Edition. Dover Books on Electrical Engineering. Dover Publications.

Babuska, R. (1998). Fuzzy Modeling for Control. International Series in Intelligent Technologies. Kluwer Academic Publishers.

Bezdek, J.C., Ehrlich, R., and Full, W. (1984). Fcm: The fuzzy c-means clustering algorithm. Computers $\&$ Geosciences, 10(2), 191-203.

FARIA, J.C.O., MUNARO, C.J., and CIARELLI, P.M. (2017). Detecção de falhas de processos não lineares via análise externa com múltiplos modelos. XIII Simpósio Brasileiro de Automação Inteligente.

Fernandes, R.T.N. (2018). Detecção de falhas em processos industriais operando em múltiplas regiões via análise externa com múltiplos modelos lineares. Dissertação de mestrado, Universidade Federal do Espírito Santo, Vitória, ES.

Filho, O.D.R. and de Oliveira Serra, G.L. (2017). Recursive fuzzy instrumental variable based evolving neurofuzzy identification for non-stationary dynamic system in a noisy environment. Fuzzy Sets and Systems. doi: https://doi.org/10.1016/j.fss.2017.05.016.

Kano, M., Hasebe, S., Hashimoto, I., and Ohno, H. (2004). Evolution of multivariate statistical process control: application of independent component analysis and external analysis. Computers and Chemical Engineering, 28, 1157-1166.

Klir, G.J. and Yuan, B. (1995). Fuzzy Sets and Fuzzy Logic: Theory and Aplications. Prentice Hall.

Mood, A.M. (1963). Introduction to the theory of statistics. Technical report.

Rotondo, D., Puig, V., Nejjari, F., and Witczak, M. (2015). Automated generation and comparison of takagi-sugeno and polytopic quasi-lpv models. Fuzzy Sets and Systems, 277, 44 - 64. doi:http://dx.doi.org/10.1016/j.fss.2015. 02.002 .

Salgado, C.M., Viegas, J.L., Azevedo, C.S., Ferreira, M.C., Vieira, S.M., and d. C. Sousa, J.M. (2017). Takagisugeno fuzzy modeling using mixed fuzzy clustering. IEEE Transactions on Fuzzy Systems, PP(99), 1-1. doi: 10.1109/TFUZZ.2016.2639565.

Takagi, T. and Sugeno, M. (1985). Fuzzy identification of systems and its applications to modeling and control. IEEE Transactions on Systems, Man and Cybernetics, SMC-15(1), 116-132. doi:10.1109/TSMC.1985.6313399.
Takane, Y. and Shibayama, T. (1991). Principal component analysis with external information on both subjects and variables. Psychometrika, 56, 97-120.

Wang, G.Z., Li, J., Hu, Y.T., Li, Y., and Du, Z.Y. (2019). Fault identification of chemical processes based on knn variable contribution and cnn data reconstruction methods. Sensors, 19(4), 929.

Wang, L.X. (1997). A Course in Fuzzy Systems and Control. Prentice Hall PTR.

Zeng, X.J. and Singh Madan, G. (1994). Approximation theory of fuzzy systems-siso case. Fuzzy Systems, IEEE Transactions on, 2(2), 162-176. doi:10.1109/91.277964. 